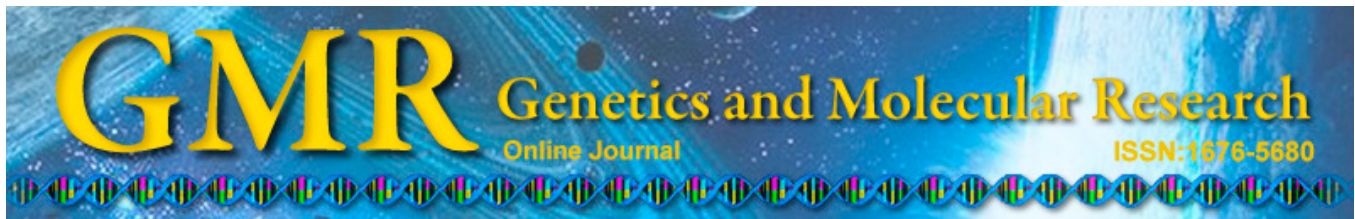

\title{
Isolation and characterization of microsatellite markers for Axonopus compressus (Sw.) Beauv. (Poaceae) using 454 sequencing technology
}

\author{
X.-L. Wang ${ }^{1 *}$, Y. Li ${ }^{2 *}$, L. Liao ${ }^{1}$, C.-J. Bai ${ }^{3}$ and Z.-Y. Wang ${ }^{1}$ \\ ${ }^{1}$ Key Laboratory of Protection and Developmental Utilization of Tropical \\ Crop Germplasm Resources of Hainan University, \\ Ministry of Education/College of Agronomy, Hainan University, Haikou, China \\ ${ }^{2}$ College of Forestry, Henan Agricultural University, Zhengzhou, China \\ ${ }^{3}$ Tropical Pasture Research Center CATAS, Danzhou, China \\ *These authors contributed equally to this study. \\ Corresponding authors: Z.-Y. Wang \\ E-mail: wangzhiyong@hainu.edu.cn
}

Genet. Mol. Res. 14 (2): 4696-4702 (2015)

Received July 15, 2014

Accepted December 3, 2014

Published May 11, 2015

DOI http://dx.doi.org/10.4238/2015.May.11.2

\begin{abstract}
Axonopus compressus (Sw.) Beauv. is a perennial herb widely used as a garden lawn grass. In this study, we used Roche 454 pyrosequencing, combined with the magnetic bead enrichment method FIASCO, to isolate simple sequence repeat markers from the A. compressus genome. A total of 1942 microsatellite loci were identified, with 53,193 raw sequencing reads. One hundred microsatellite loci were selected to test the primer amplification efficiency in 24 individuals; 14 primer pairs yielded polymorphic amplification products. The number of observed alleles ranged from two to six, with an average of 3.5. Shannon's Information index values ranged from 0.169 to 0.650 , with an average of 0.393 . Nei's genetic diversity values ranged from 0.108 to 0.457 , with an average of 0.271 .
\end{abstract}


This first set of microsatellite markers developed for Axonopus will assist in the development of molecular marker-assisted breeding and the assessment of genetic diversity in A. compressus.

Key words: Microsatellite markers; Genetic diversity; Roche 454 sequencing; Axonopus compressus

\section{INTRODUCTION}

Axonopus compressus (Sw.) Beauv. (Poaceae) is a perennial herb native to South America. In China, the wild germplasm resources of Axonopus compressus are mainly distributed in the tropical and subtropical climate zones, such as Hainan, Fujian, Guangdong, Guangxi, Yunnan, and Guizhou (Liao et al., 2011). All A. compressus individuals are polyploidy, with $2 \mathrm{n}=4 \mathrm{X}=40,2 \mathrm{n}=5 \mathrm{X}=50$, and $2 \mathrm{n}=6 \mathrm{X}=60$ chromosomes (Delay, 1950). Because of its low height, easy propagation, fast growth, good elasticity, and strong adaptability, A. compressus has been widely used as a warm-season garden lawn grass (Zhang and Xi, 2005; Zhou et al., 2005). Previous studies of $A$. compressus have mainly focused on germplasm investigation and its physiological characteristics (Jian and Zhou, 2003; Guo et al., 2004; Xi et al., 2004a,b; Huang et al., 2012); however, the genetic diversity of its wild germplasm resources is little known. Therefore, developing a set of reliable and informative DNA markers to aid in assessing the genetic diversity of its wild germplasm resources would be beneficial for the development of its breeding program.

Microsatellites, or simple sequence repeats (SSRs), are ubiquitous in eukaryotic genomes (Zhang et al., 2010), and have become increasingly popular in genetic studies because of their hypervariability, abundance, reproducibility, ease of scoring, and fast throughput (Sun et al., 2008). Fast isolation by amplified fragment length polymorphism (AFLP) of sequences containing repeats (FIASCO) has been proven to be the most effective magnetic bead enrichment method for isolating SSR markers (Sun et al., 2008; Yang et al., 2009; Hou et al., 2011; Wang et al., 2014a). However, this method is laborious and difficult to use, and cannot obtain a large number of SSRs in one experiment (Wang et al., 2014b). Next-generation sequencing (NGS) has become a more efficient approach to generate superior resources for the development of SSR markers (Kumar et al., 2014; Huang et al., 2014). To date, the most common isolation SSR methods using NGS have been transcriptome sequencing and shotgun sequencing (Jenkins et al., 2013; Bai et al., 2014; Kumar et al., 2014; Huang et al., 2014). In this study, we used Roche 454 pyrosequencing, combined with the magnetic bead enrichment method FIASCO, to isolate SSR markers from the A. compressus genome.

\section{MATERIAL AND METHODS}

\section{Isolation of microsatellite markers}

Genomic DNA was extracted using a plant genomic DNA kit (Tiangen Biotech, Beijing, China) from silica gel-dried leaves, according to the manufacturer protocol. Approximately $1 \mu \mathrm{g}$ of genomic DNA was used to generate a shotgun library, following the 454 
Roche protocol. The shotgun library was further enriched by eight 5'-biotinylated probes: (AG)10, (AC)10, (AAC)8, (ACG)8, (AAG)8, (AGG)8, (ACAT)6, and (ATCT)6, according to the protocol of $\mathrm{Li}$ et al. (2007). The enriched products were subsequently sequenced on one 16th of a picotiter plate using a Roche 454 GS-FLX+ System (Shanghai, China). Microsatellite searching was performed using MISA (http://pgrc.ipk-gatersleben.de/misa/), search parameters were set as ten repeat units for mononucleotides, six repeat units for dinucleotides, five repeat units for tri-, tetra-, penta-, and hexanucleotides, and the maximum interruption between two SSRs to consider a SSR as a compound was set at 100 nucleotides. Primer designing was conducted using Primer3 (Rozen and Skaletsky, 2000), and the polymerase chain reaction (PCR) product size range was set at 100-400 bp; the remaining parameters were set at default values.

\section{PCR amplification and genotyping}

A total of 24 individuals of $A$. compressus from China and Australia (Table 1) were used to analyze the polymorphisms of the microsatellite primers. PCRs were performed in a total volume of $30 \mu \mathrm{L}$, which contained $30 \mathrm{ng}$ of genomic DNA, $0.2 \mathrm{mM} / \mathrm{L}$ of each dNTP, 0.3 $\mu \mathrm{M} / \mathrm{L}$ of each primer, $3 \mu \mathrm{L}$ of $10 \mathrm{X}$ polymerization buffer, and 1 unit of Taq polymerase (Tiangen Biotech, Beijing, China), using a PTC-200 Thermal Cycler (MJ Research, Watertown, MA, USA). The PCR was conducted as follows: an initial denaturation for $5 \mathrm{~min}$ at $95^{\circ} \mathrm{C}$, followed by 35 cycles of denaturation for $30 \mathrm{~s}$ at $94^{\circ} \mathrm{C}$, annealing for $30 \mathrm{~s}$ at $48-60^{\circ} \mathrm{C}$ (optimized for each locus, Table 2), extension for $40 \mathrm{~s}$ at $72^{\circ} \mathrm{C}$, and a final extension step of $8 \mathrm{~min}$ at $72^{\circ} \mathrm{C}$. The PCR products were electrophoresed on an $8 \%$ native polyacrylamide gel and visualized by silver staining. Allele sizes were estimated using a 100-bp DNA ladder (Takara, Dalian, Liaoning, China) as a reference.

\section{Data analysis}

Polymorphism statistics, including allele number, Nei's measure of gene diversity (Nei, 1973), and Shannon's Information index, were estimated using POPGENE v.1.3.2 (Yeh et al., 2000). Genetic relationships between 24 individuals of A. compressus, based on Nei's genetic distance (Nei, 1978), were examined using the unweighted pair group method with arithmetic mean (UPGMA) in POPGENE (Yeh et al., 2000).

\section{RESULTS AND DISCUSSION}

A total of 53,193 raw sequencing reads were obtained. Of these, 9735 sequences (18.30\%) contained SSRs and only 1942 sequences (3.65\%) were fit for primer pair design. To test the primer amplification efficiency, we randomly selected 100 microsatellite sequences to design primer pairs, and deposited these sequences in GenBank (KM110835KM110934). Of the 100 primer pairs, 66 primer sets were abandoned because of the unsuccessful amplification of target fragments. The remaining 34 primer pairs were tested for polymorphisms in 24 individuals from China and Australia (Table 1); 14 loci displayed 
polymorphisms and 20 were monomorphic. The genetic diversity parameters of each population are presented in Table 2. The number of observed alleles ranged from two (Ac 019, $A c$ 021, and $A c$ 094) to six (Ac 087), with an average of 3.5. Shannon's Information index values ranged from 0.169 ( $A c$ 067) to 0.650 ( $A c$ 091), with an average of 0.393 . Nei's genetic diversity values ranged from 0.108 ( $A c$ 067) to 0.457 (Ac 091), with an average of 0.271 . As with other polyploidy species, traditional measures of genetic variability, such as standard tests for deviations from the Hardy-Weinberg equilibrium and linkage disequilibrium, could not be determined in $A$. compressus because the exact copy number for each locus is currently unknown (Huang et al., 2009). The UPGMA tree indicated that individuals In 1-In 8, In 11-In 12, In 14-In 15, and In 18-In 22 had close phylogenetic relationships, and In 9-In 10, In 13, In 16-In 17, and 23-24 were more closely related (Figure 1). One individual from Australia was most closely related to an individual from Dingan, China. The identification of genetic relationships between individuals from different geographical regions will assist in parent selection for crossbreeding. This first set of microsatellite markers developed for Axonopus is important in the development of molecular marker-assisted breeding and the assessment of wild germplasm resources, in terms of genetic diversity, in A. compressus.

Table 1. Details of sample locations of Axonopus compressus.

\begin{tabular}{llcc}
\hline Individual No. and code & Location & Latitude & Longitude \\
\hline In 1 & Danzhou, Hainan & 19.30 & 109.29 \\
In 2 & Dingan, Hainan & 19.41 & 110.20 \\
In 3 & Dingan, Hainan & 19.33 & 110.16 \\
In 4 & Danzhou, Hainan & 19.30 & 109.29 \\
In 5 & Baisha, Hainan & 19.13 & 109.26 \\
In 6 & Qionghai, Hainan & 19.21 & 110.41 \\
In 7 & Sanya, Hainan & 17.27 & 109.20 \\
In 8 & Wenchang, Hainan & 18.30 & 109.34 \\
In 9 & Wanning, Hainan & 18.42 & 110.23 \\
In 10 & Guigang, Guangxi & 22.59 & 109.36 \\
In 11 & Chengmai, Hainan & 19.75 \\
In 12 & Qiongzhong, Hainan & 19.24 & 109.93 \\
In 13 & Ledong, Hainan & 18.81 & 109.84 \\
In 14 & Danzhou, Hainan & 19.30 & 109.17 \\
In 15 & Haikou, Hainan & 19.55 & 109.29 \\
In 16 & Menghai, Yunnan & 21.41 & 110.14 \\
In 17 & Ledong, Hainan & 18.44 \\
In 18 & Yingde, Guangdong & 24.13 & 102.02 \\
In 19 & Canberra, Australia & 35.29 & 108.51 \\
In 20 & Hekou, Yunnan & 22.55 & 113.50 \\
In 21 & Ceheng, Guizhou & 24.59 & 149.13 \\
In 22 & Zhangpu, Fujian & 24.08 & 103.36 \\
In 23 & Wuzhou, Guangxi & 23.41 & 105.46 \\
In 24 & Boluo, Guangdong & 23.18 & 117.46 \\
\hline & & 115.25 \\
& & 114.28 \\
\hline
\end{tabular}




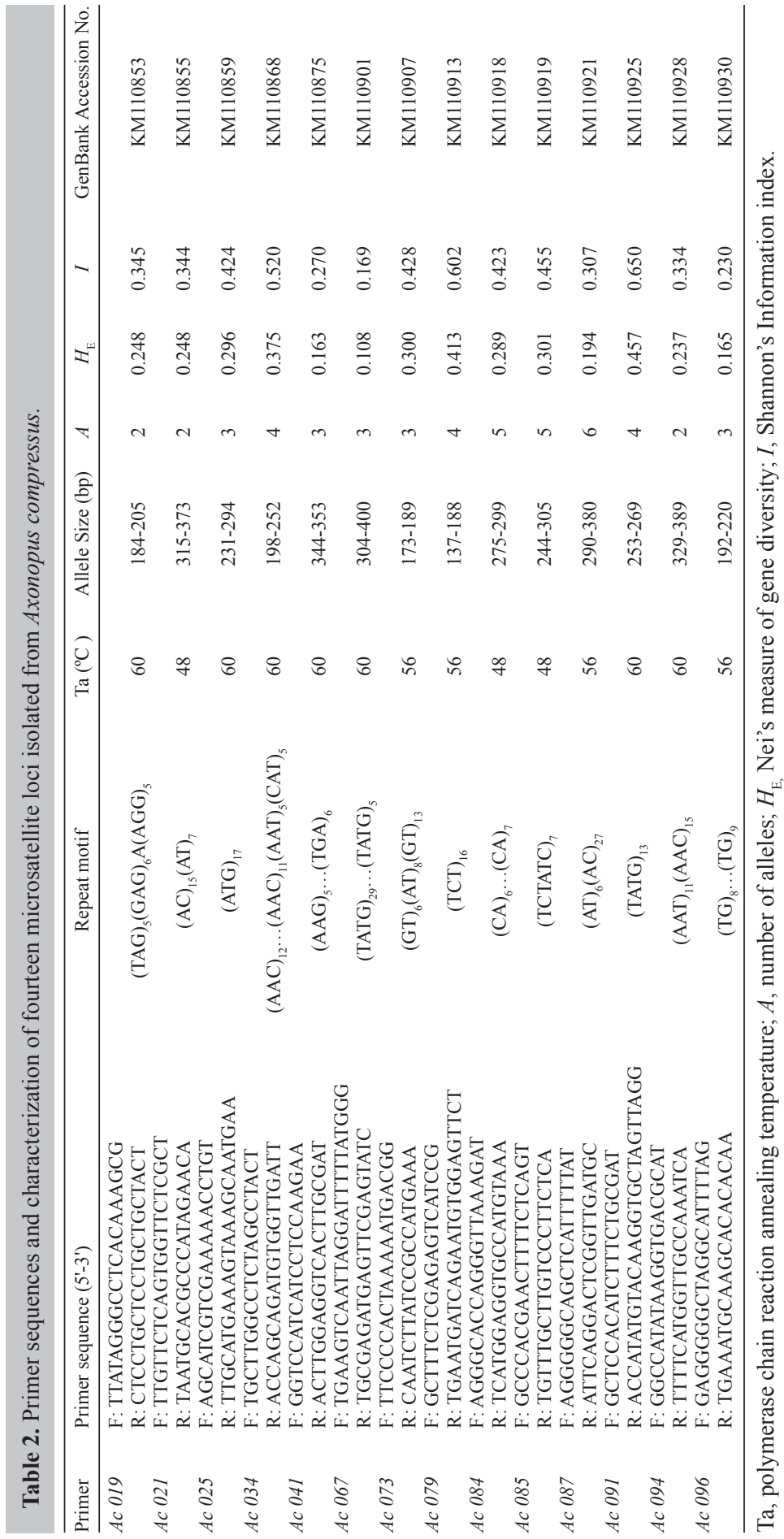




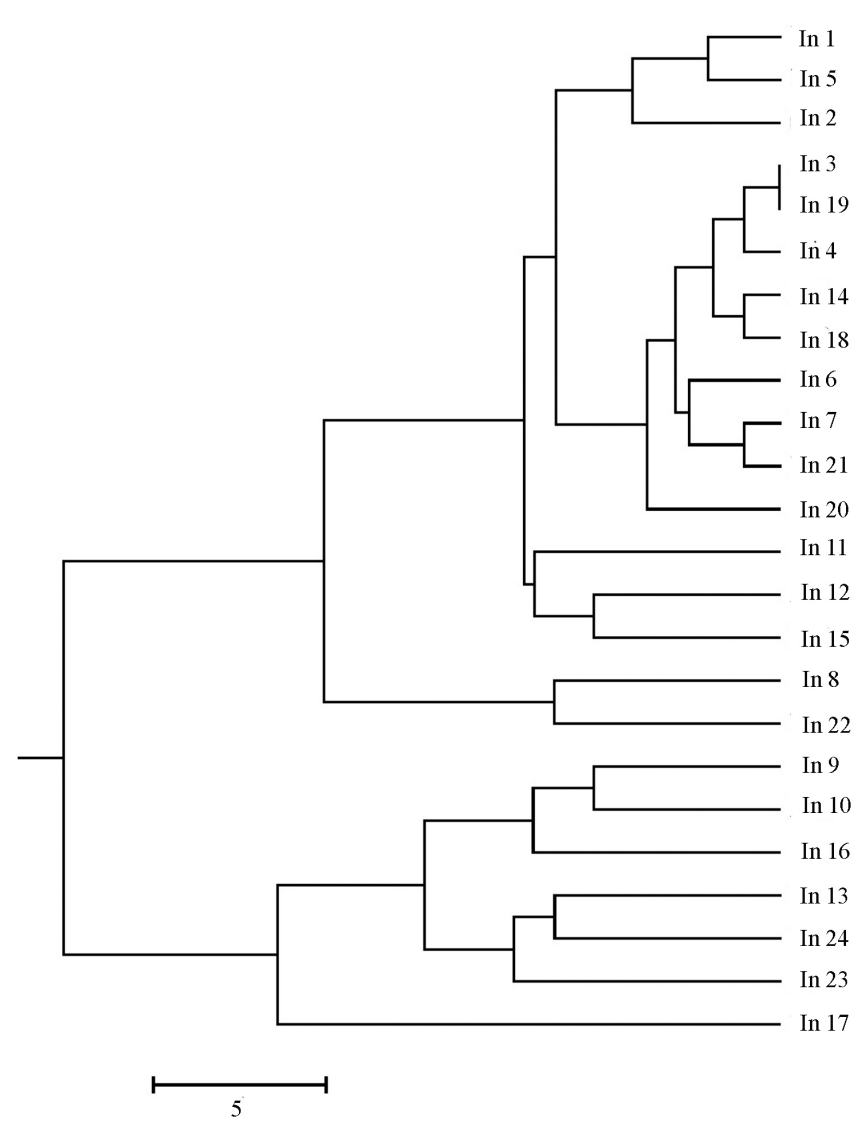

Figure 1. Unweighted pair group method with arithmetic mean (UPGMA) tree illustrating the genetic relationships between 24 individuals of Axonopus compressus, based on Nei's genetic distance.

\section{ACKNOWLEDGMENTS}

Research supported by the National Natural Science Foundation of China (\#31060266, \#31260489 and \#31100272) and the Natural Science Foundation of Hainan (\#310031).

\section{REFERENCES}

Bai TD, Xu LA, Xu M and Wang ZR (2014). Characterization of masson pine (Pinus massoniana Lamb.) microsatellite DNA by 454 genome shotgun sequencing. Tree Genet. Genomes 10: 429-437.

Delay C (1950). Nombres chromosomiques chez les Phanrogames. Rev. Cytol. et Biol. Veg. 12: 1-368.

Guo LH, Wang L, Liu JX and Yang YF (2004). A study on photosynthetic physiological characters of vegetative and reproductive tillers of Axonopus compressus. Acta Agres. Sin. 12: 103-106.

Hou XG, Guo DL, Cheng SP and Zhang JY (2011). Development of thirty new polymorphic microsatellite primers for Paeonia suffruticosa. Biol. Plantarum 55: 708-710.

Huang DN, Zhang YQ, Jin MD, Li HK, et al. (2014). Characterization and high cross-species transferability of microsatellite markers from the floral transcriptome of Aspidistra saxicola (Asparagaceae). Mol. Ecol. Resour. 14: 569-577.

Huang XH, Liao L, Bai CJ and Wang ZY (2012). Study on screening of salt concentration gradient and critical concentration on Axonopus compressus. Pratacul. Sci. 29: 599-604. 
Huang Y, Li Y, Hu X, Ge XJ, et al. (2009). Development of twelve polymorphic microsatellite loci in polyploid endangered Omphalogramma vincaeflora Franch. (Primulaceae). Conserv. Genet. 10: 515-517.

Jenkins TM, Eaton TD, Nunziata SO, Lance SL, et al. (2013). Paired-end illumina shotgun sequencing used to develop the first microsatellite primers for Megacopta cribraria (F.) (Hemiptera: Heteroptera: Plataspidae). J. Entomol. Sci. 48: 345-351.

Jian HY and Zhou SQ (2003). The photosynthetic characteristics in leaves of carpet grass-Axonopus compressus. Guihaia 23: 181-184.

Kumar S, Shah N, Garg V and Bhatia S (2014). Large scale in-silico identification and characterization of simple sequence repeats (SSRs) from de novo assembled transcriptome of Catharanthus roseus (L.) G. Don. Plant Cell Rep. 33: 905-918.

Li Y, Li LF, Chen GQ and Ge XJ (2007). Development of ten microsatellite loci for Gentiana crassicaulis (Gentianaceae). Conserv. Genet. 8: 1499-1501.

Liao L, Huang XH, Bai CJ and Wang ZY (2011). Study on responses of Axonopus compressus (Sw.) Beauv. to aluminum stress and critical aluminum concentration. Chin. J. Trop. Crops 32: 1235-1239.

Nei M (1973). Analysis of gene diversity in subdivided populations. Proc. Natl. Acad. Sci. USA 70: 3321-3323.

Nei M (1978). Estimation of average heterozygosity and genetic distance from a small number of individuals. Genetics 76: 379-390.

Rozen S and Skaletsky H (2000). Primer3 on the WWW for general users and for biologist programmers. In: Bioinformatics methods and protocols: methods in molecular biology (Krawetz S and Misener S, eds.) Humana Press, Totowa, USA, pp. 365-386.

Sun QB, Li LF, Li Y, Wu GJ, et al. (2008). SSR and AFLP markers reveal low genetic diversity in the biofuel plant Jatropha curcas in China. Crop Sci. 48: 1865-1871.

Wang M, Xue F, Yang P, Duan XY, et al. (2014a). Development of SSR markers for a phytopathogenic fungus, Blumeria graminis f.sp tritici, using a FIASCO protocol. J. Integr. Agr. 13: 100-104.

Wang W, Li Z and Li Y (2014b). Isolation and characterization of microsatellite markers for Cotinus coggygria Scop. (Anacardiaceae) by 454 pyrosequencing. Molecules 19: 3813-3819.

Xi JB, Chen P, Liu CC, Zheng YZ, et al. (2004a). Study on morphological and biological variation of different wild carpetgrass. J. Sichuan Grassland 12: 15-19.

Xi JB, Chen P, Zheng YZ and Yang ZY (2004b). An investigation of Axonopus compressus germplasm resources in China. Acta Prata. Sin. 13: 52-57.

Yang JB, Yang J, Li HT, Zhao Y, et al. (2009). Isolation and characterization of 15 microsatellite markers from wild tea plant (Camellia taliensis) using FIASCO method. Conserv. Genet. 10: 1621-1623.

Yeh FC, Yang R, Boyle TJ and Xiyan JM (2000). PopGene 32. Microsoft Window-based freeware for population genetic analysis, version 1.32. Molecular Biology and Biotechnology Centre, University of Alberta, Edmonton, Canada.

Zhang HX and Xi JB (2005). Study on the germplasm resource and ecological characteristics of wild carpetgrass (Axonopus compressus) in Guangdong. Grassland Turf. 6: 25-27.

Zhang MS, Wang H, Dong ZY, Qi B, et al. (2010). Tissue culture-induced variation at simple sequence repeats in sorghum (Sorghum bicolor L.) is genotype-dependent and associated with down-regulated expression of a mismatch repair gene, MLH3. Plant Cell Rep. 29: 51-59.

Zhou YL, Zhang XQ and Liu W (2005). Research progress of Axonopus compressus. J. Sichuan Grassland 11: 24-26. 